\title{
CAMA
}

Centre for Applied Macroeconomic Analysis

\section{Dynamic Cost of Living Index for Storable Goods}

\section{CAMA Working Paper 88/2019 December 2019}

\section{Kozo Ueda}

Waseda University

Centre for Applied Macroeconomic Analysis, ANU

\section{Abstract}

Consumers hold inventory for future uses. This study investigates how such intertemporal decisions influence the cost-of-living index (COLI). To this end, I construct a simple dynamic model, in which goods are storable and nonresalable, and prices take either high (regular price) or low values (sales). I then introduce two types of dynamic COLIs. Simulation results show that neither index satisfies both monotonicity and the time reversal test. 


\section{Keywords}

consumer inventory, cost-of-living index, price index

\section{JEL Classification}

C43, D15, E31

\section{Address for correspondence:}

(E) cama.admin@anu.edu.au

\section{ISSN 2206-0332}

The Centre for Applied Macroeconomic Analysis in the Crawford School of Public Policy has been established to build strong links between professional macroeconomists. It provides a forum for quality macroeconomic research and discussion of policy issues between academia, government and the private sector.

The Crawford School of Public Policy is the Australian National University's public policy school, serving and influencing Australia, Asia and the Pacific through advanced policy research, graduate and executive education, and policy impact. 


\title{
Dynamic Cost of Living Index for Storable Goods
}

\author{
Kozo Ueda*
}

December 10, 2019

\begin{abstract}
Consumers hold inventory for future uses. This study investigates how such intertemporal decisions influence the cost-of-living index (COLI). To this end, I construct a simple dynamic model, in which goods are storable and nonresalable, and prices take either high (regular price) or low values (sales). I then introduce two types of dynamic COLIs. Simulation results show that neither index satisfies both monotonicity and the time reversal test.
\end{abstract}

JEL Classification Numbers: C43, D15, E31

Keywords: consumer inventory; cost-of-living index; price index

\section{Introduction}

The cost-of-living index (COLI) serves as an important measure of price index, having strong theoretical foundations (Könus,1924). However, most models are static, focusing on intratemporal substitutions among goods and neglecting intertemporal ones despite the fact that most goods are storable.

In this note, I study the properties of the dynamic COLI. To this end, I construct a simple model of storable goods, in which storable goods are nonresalable and households optimize how much they purchase, consume, and stockpile. Prices take either high or low values exogenously. I then numerically solve for model equilibrium using parameter values calibrated to the Japanese economy. The dynamic COLI is defined as a welfare measure for compensating variation in response to price changes, as in Könus (1924), but welfare is now calculated based on lifelong utility, not period utility. An issue here is that welfare depends on a state variable, a household inventory, which is endogenous in the previous period. When comparing the dynamic COLI between two periods, it is a matter of judgment what level of household inventory we should condition. As such, I examine two definitions for the dynamic COLI.

\footnotetext{
${ }^{*}$ Waseda University and Centre for Applied Macroeconomic Analysis (CAMA) (E-mail: kozo.ueda@waseda.jp). I would like to thank Kota Watanabe and Tsutomu Watanabe for the discussions. This research was funded by the JSPS Grant-in-Aid for Scientific Research (16KK0065) and Nomura Foundation (N19-2-E40). All remaining errors are my own.
} 
I find that the dynamic COLI is difficult to use for practical purposes as it does not satisfy the following two basic properties simultaneously, which are considered as essential in price indexes: monotonicity and the time reversal test (see ILO et al. 2004). One type of the dynamic COLI satisfies monotonicity, exhibiting an increase (decrease) in the dynamic COLI when the prices of storable goods increase (decrease). However, it does not satisfy the time reversal test. The price index does not revert to the same level, although the prices of storable goods revert to the same level. By systematically accumulating the COLI change whenever temporary sales occur, a chain drift is generated in the long run. Another type of the dynamic COLI satisfies the time reversal test, but not monotonicity. This unsatisfactory result poses a challenge to both economists and practitioners. Until we solve this problem, the use of the conventional static COLI may be better advised, because it not only satisfies both monotonicity and the time reversal test, but is also simple to understand and construct.

Reis (2009) and Osborne (2018) propose a dynamic price index that considers the intertemporal decisions of households. In Reis (2009), the dynamic COLI is constructed from the compensating variation of lifelong utility (welfare) rather than period utility. The main difference is that, although storable goods exist under both models, they are irreversible in my model. Once households purchase storable goods, they cannot sell them to others and must consume them by themselves, which is the key feature of most storable goods and becomes a source of asymmetry (chain drift). Consequently, welfare cannot be expressed as a function of wealth including inventory, which makes us unable to use the dynamic COLI defined in Reis (2009). ${ }^{1}$

Osborne (2018) considers irreversible storable goods like our study, and computes the dynamic COLI following Gowrisankaran and Rysman (2012). The COLI in his definition is essentially the one that keeps period utility, not lifelong utility, constant over time, although it takes account of agents' dynamic optimization problem. Furthermore, Osborne (2018) discards the chained price index claiming it is not theoretically correct or is subject to excessive volatility, while this study considers the chained price index seriously (see, for example, Ivancic, Diewert, and Fox 2011 for the same standpoint). This study investigates the short-run properties of the dynamic COLI. This aspect is almost always neglected but important, because intertemporal substitutions (stockpiling) occur over a short horizon, of days. How the dynamic COLI fluctuates when the prices of storable goods change is of particular concern here.

\footnotetext{
${ }^{1}$ As the second difference, in the proposed model, households receive utility from consuming storable goods, while in Reis' (2009), households receive utility by holding their stock (i.e., goods are durable). Third, the model does not have risk-free bonds as a medium of savings or borrowings. Therefore, households cannot save, except for storable goods, which is introduced into this model for simplicity. In Reis' (2009) model, storable goods are essentially similar to risk-free bonds in providing a medium of savings and borrowings, except that the former directly contributes to utility.
} 
On the stockpiling of storable goods and its implications for the price index, many studies have already been published, such as Boizot, Robin, and Visser (2001), Erdem, Imai, and Keane (2003), Feenstra and Shapiro (2003), Hendel and Nevo (2006a,b), Gowrisankaran and Rysman (2012), Wang (2013), and Osborne (2018) among others. Feenstra and Shapiro (2003) report a large drift in the orthodox Törnqvist price index, attribute it to the stockpiling of storable goods, and propose a modified fixed-base price index.

\section{Model}

\section{$2.1 \quad$ Model Setup}

The model is similar to that of Hendel and Nevo (2006a). A representative household (HH) maximizes its welfare $W_{t}$ in each period $t$ as

$$
W_{t}=\mathbb{E}_{t} \sum_{i=0}^{\infty} \beta^{t-1} u\left(c_{t+i}, c_{t+i}^{N}\right),
$$

where $0<\beta \leq 1$ represents a discount factor; $C_{t}$ and $C_{t}^{N}$ represent the consumption of storable and non-storable goods, respectively; and function $u\left(c_{t}, c_{t}^{N}\right)$ satisfies $u_{c}>0, u_{c^{N}}>0, u_{c c}<0$, $u_{c^{N} c^{N}}<0$, and the Inada condition. The HH purchases storable goods by $x_{t}$. The HH holds the storable goods as

$$
i_{t}=i_{t-1}-c_{t}+x_{t}
$$

where $i_{t}$ is the inventory at the end of period $t$. The non-storable goods are purchased by $c_{t}^{N}$ and consumed by the same amount in period $t$. Nonnegative constraints for $x_{t}$ and $i_{t}$ are the key property of most storable goods. The storable goods cannot be sold in the second market, once the $\mathrm{HH}$ purchases them. The HH does not have a savings opportunity, except for the inventory of storable goods. I neglect the depreciation of storable goods, the cost of holding inventory, and the discreteness of the unit of purchase without generality loss.

The budget constraint is expressed as

$$
p_{t} x_{t}+c_{t}^{N}=1
$$

where $p_{t}$ represents the price of storable goods and the price of non-storable goods is normalized to one. The HH receives the endowment of one. For timing, in each period, the HH holds $i_{t-1}$, observes $p_{t}$, purchases $x_{t}$ and $c_{t}^{N}$, and then consumes $c_{t}$ and $c_{t}^{N}$, which determines $i_{t}$.

The price of storable goods takes either of the two $p_{H}$ or $p_{L}$, where $p_{H}>p_{L}$. The price 
evolves according to the Markov process as

$$
\begin{aligned}
& \operatorname{Prob}\left(p_{L} \mid p_{H}\right)=\bar{q} \\
& \operatorname{Prob}\left(p_{L} \mid p_{L}\right)=\underline{q},
\end{aligned}
$$

where $0<\underline{q}, \bar{q}<1$.

The value function is given by

$$
V\left(i_{t-1}, p_{t}\right)=\max _{c_{t}, c_{t}^{N}, x_{t}, i_{t}}\left[u\left(c_{t}, c_{t}^{N}\right)+\beta \mathbb{E}_{t} V\left(i_{t}, p_{t+1}\right)\right]
$$

subject to equations (2)-(4). Equivalently, it can be rewritten as

$$
V\left(i_{t-1}, p_{t}\right)=\max _{x_{t}, i_{t}}\left[u\left(i_{t-1}+x_{t}-i_{t}, 1-p_{t} x_{t}\right)+\beta \mathbb{E}_{t} V\left(i_{t}, p_{t+1}\right)\right],
$$

subject to $x_{t} \geq 0, i_{t} \geq 0$, and equation (4).

\subsection{Dynamic COLI}

Könus (1924) defined the COLI as the welfare measure for compensating variation in response to price changes. To extend this to a dynamic model, I refer to Reis (2008), Gowrisankaran and Rysman (2012), and Osborne (2018), and introduce the following two definitions for the dynamic COLI in the presence of storable goods:

(i) I define compensating variation $P_{t}^{W}$ so that

$$
V\left(i_{t-1}, p_{t}, P_{t}^{W}\right)=V\left(i_{t-2}, p_{t-1}, 1\right)
$$

or $V\left(i_{t-1}, p_{t}, P_{t}^{W}\right)=V_{0}$, where $V_{0}$ is a certain constant and $V\left(i_{t-1}, p_{t}, P_{t}^{W}\right)$ is defined as

$$
V\left(i_{t-1}, p_{t}, P_{t}^{W}\right) \equiv \max _{x_{t}, i_{t}}\left[u\left(i_{t-1}+x_{t}-i_{t}, P_{t}^{W}-p_{t} x_{t}\right)+\beta \mathbb{E}_{t} V\left(i_{t}, p_{t+1}, 1\right)\right]
$$

The endowment of $P_{t}^{W}$, which is transitory, is chosen to achieve constant welfare over time. Then, the change in the dynamic COLI from the previous period, $\pi_{t}^{W}$, equals $\pi_{t}^{W}=\log \left(P_{t}^{W}\right)$.

This definition resembles that introduced in Gowrisankaran and Rysman (2012) and Osborne (2018). A main difference is that, in this study, the dynamic COLI is the unexpected one-time compensation that holds welfare (lifelong utility) constant over time. In the abovecited studies, it is a state-contingent compensation that holds period utility constant over time. This difference is considered to matter when a price decreases. At sales, households sacrifice today's period utility, because goods are stockpiled for the future. In such a case, a large posi- 
tive compensation might be needed to hold period utility constant, although the occurrence of sales increases its welfare and, thus, a negative compensation (tax) is needed to keep welfare constant.

(ii) Whereas state variable $i_{t}$ varies over time, you may think natural to compare welfare given the same amount of inventory. Therefore, I introduce alternative $P_{t}^{W}$ so that

$$
V\left(i_{t-2}, p_{t}, P_{t}^{W}\right)=V\left(i_{t-2}, p_{t-1}, 1\right)
$$

where I base the inventory at the end of period $t-2$.

It should be noted that, in Reis' (2009) model, all goods are resalable in the second market, and thus, welfare is expressed by households' wealth at the beginning of each period, which includes $i_{t-1}$ (inventory) times $p_{t}$ (the price of the goods in the current period). By contrast, welfare in my model depends on state variable $i_{t-1}$, not $p_{t} i_{t-1}$. Thus, we cannot consider compensation for wealth as in Reis (2009).

\section{$3 \quad$ Numerical Simulation}

\subsection{Equilibrium}

The Bellman equation (5) characterizes the optimal behavior of the HH. To solve for equilibrium, I modify the code developed by Nakamura and Steinsson (2010) and take the following steps. First, I specify a finite grid of points for the state variables, $i_{t-1}, p_{t}$, as well as $P_{t}^{W}$. Second, I solve for HH's policy functions for $x_{t}$ and $i_{t}$ by iterating equation (5).

\section{Parameterization}

The unit of time is a day. I assume $u\left(c_{t}, c_{t}^{N}\right)=\alpha \log \left(c_{t}\right)+(1-\alpha) \log \left(c_{t}^{N}\right)$, where I set the share of storable goods $\alpha$ at 0.1 . Discount factor $\beta$ equals $0.96^{1 / 365}$. Regarding the price, I set the values to be consistent with Japanese retailers' scanner data in the 2000s and 2010s: price $p_{L}$ is set at 0.90 , while $p_{H}$ equals the price of non-storable goods, that is, 1 , and the probability of sales is given by $\bar{q}=0.03$ and $\underline{q}=0.50 .^{2}$

\section{Policy Functions}

Before studying the COLI, I verify the equilibrium. Figure 1 shows the policy functions for $c_{t}$, $x_{t}$, and $i_{t}$ and welfare $V_{t}$, given $i_{t-1}$. Welfare $V_{t}$ is expressed by the inverse of the compensating

\footnotetext{
${ }^{2}$ See Sudo, Ueda, and Watanabe (2014) and Sudo et al. (2018) for the detailed data descriptions. For the numerical simulation, $x$ is discretized by 0.01 , which is sufficiently small compared with the average consumption of about 0.1 .
} 
variation of current income, where it is normalized to zero when $i_{t-1}=0$ and $p_{t}=p_{H}$. For example, 0.1 means that an increase in welfare corresponds to a 10 percent increase in current income.

The results are simple to interpret. Consumption, as well as welfare, are higher at a low price than at a high price and nondecreasing with the previous inventory. The amount of purchases is also higher at a low price than at a high price. Opposite to consumption and welfare, the amount of purchases is nonincreasing with the previous inventory, because there is a lower need for stockpiling. The targeted level of inventory is nondecreasing with the previous inventory. This suggests that consumption is not constant. It is low when household inventory is low or the price high. Additionally, it is important to note that welfare becomes less sensitive to price (whether high or low) when inventory is high, because there is almost no need to purchase when the price is high.
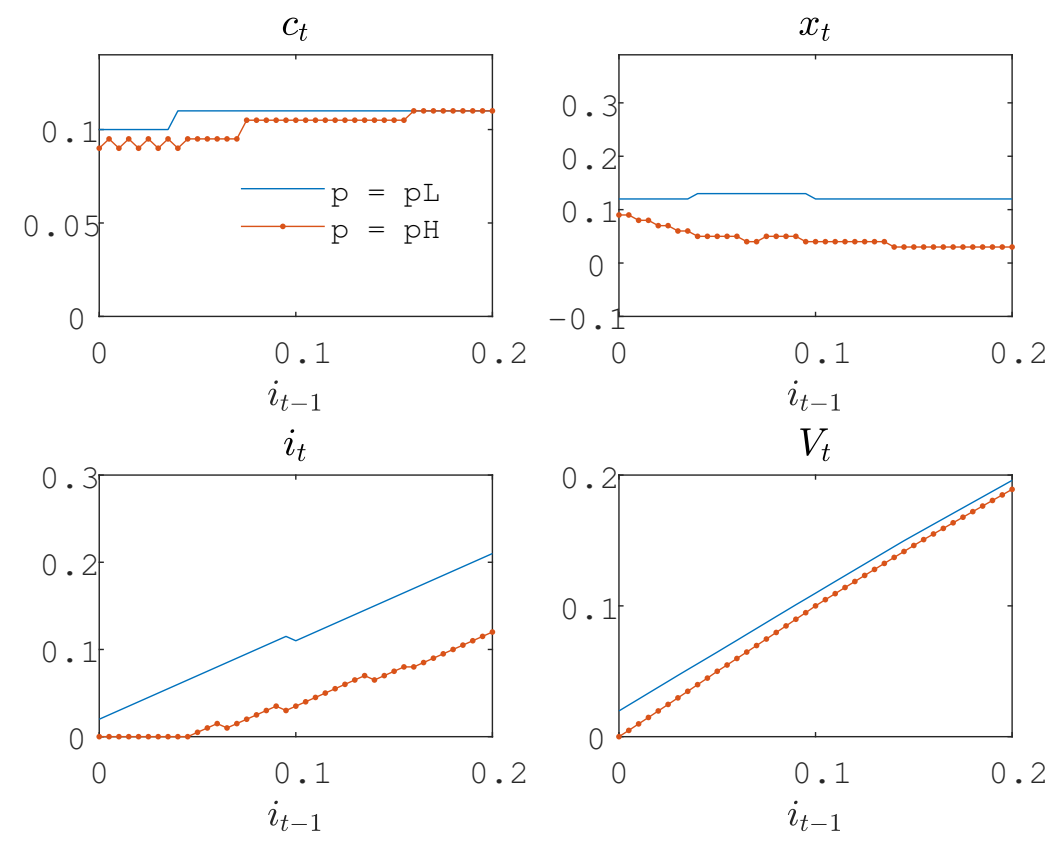

Figure 1: Policy Functions and Welfare

\subsection{Monotonicity of the Dynamic COLI}

Next, I study the properties of the dynamic COLI. To do this, I generate the time series of prices for 21 years $(T=365 \times 21)$ and compute endogenous variables in each period, discarding the first 365 samples. The top two panels of Figure 2 represent the scatter plots for the changes in the dynamic COLI $\left(\pi_{t}^{W}\right)$ on the vertical axis with the changes in the prices of storable goods $\left(\Delta \log \left(p_{t}\right)\right)$ on the horizontal axis. Each dot indicates an observation in each period $t$. 
Additionally, Table 1 shows the means and standard deviations of the changes in the dynamic COLIs.

The top left-hand panel of Figure 2 shows that the dynamic COLI based on definition (i) does not necessarily increase (decrease) when the prices of storable goods increase (decrease), although the correlation coefficient is positive (0.24). Even when prices remain unchanged, the dynamic COLI fluctuates. To understand why, I represent inventories $i_{t-1}$ and $i_{t-2}$ and purchase $x_{t}$ in the bottom panels. They show that $i_{t-1}$ tends to be large when prices increase (positive $\Delta \log \left(p_{t}\right)$ ), whereas there is no significant relationship between $i_{t-2}$ and $\Delta \log \left(p_{t}\right)$. Since the price increase in period $t$ suggests that prices were low in period $t-1$, the level of inventory at the end of period $t-1$ tends to be large. This decreases the need for purchases (low $x_{t}$ ) and increases welfare (high $V_{t}$ ) and, in turn, contributes to almost no increase in the dynamic COLI, despite the increase in $p_{t}$. The opposite is also true. Inventory $i_{t-1}$ tends to be low when prices decrease, because this implies that past prices were high. Therefore, welfare and the dynamic COLI tend to be low and high, respectively. Such a nonmonotonic property is difficult to use for a practical objective.

Conversely, the dynamic COLI based on definition (ii) holds a monotonic and positive relationship with price changes. The dynamic COLI increases, when prices of storable goods increase, and vice versa. When prices remain unchanged from the previous period, the dynamic COLI does not change. This property is obvious from equation (8). As a result, $\pi_{t}^{W}$ and $\Delta \log \left(p_{t}\right)$ have a correlation as high as 1.00 .

\subsection{Time Reversal of the Dynamic COLI}

However, the dynamic COLI based on definition (ii) suffers from bias. Figure 3.4 shows the time series of the levels of the dynamic COLIs, constructed by making a chain of their changes $\Pi_{j=1}^{J}\left(1+\pi_{t+j}^{W}\right)$ for $J=1,2, \cdots, 7300$. The right-hand panel shows that the price index decreases by 3 percent over two decades. The average inflation rate of the dynamic COLI is only -4.00 . $10^{-6}$ annually, but accumulating this leads to a clear price decrease.

There are two counteracting forces behind this chain drift. On one hand, welfare is more sensitive to price changes when inventory is low than when it is high. Furthermore, when $i_{t-2}$ is high, the probability of $p_{t-2}=p_{L}$ is higher than the unconditional probability of being $p_{L}$, because the $\mathrm{HH}$ should purchase storable goods for stockpiling. Since the price takes only two values and has an inertia, price $p_{t}$ is more likely to increase from $p_{L}$ to $p_{H}$ than decrease. Therefore, the correlation between $i_{t-2}$ and $\Delta \log \left(p_{t}\right)$ becomes positive. This works to decrease the dynamic COLI. On the other hand, given the same inventory level, the size of the changes in the dynamic COLI tends to be larger when $p_{t}$ increases than when $p_{t}$ decreases. Put differently, 
because of the nonlinearity of $i_{t}$ and $x_{t}$, the HH dislikes price increases more than prefers price decreases. This generates an upward bias. In the simulation, the former force dominates, yielding around the 3 percent downward drift over two decades. Although the size of the drift does not seem extraordinarily large, this size becomes about ten times larger if all goods are storable, since the model assumes that only ten percent of goods are storable (i.e., $\alpha=0.1$ ). Moreover, I show below that different, but plausible, parameter values yield far larger chain drift.

The advantage of definition (i) is that it does not have such a bias. The left-hand panel in Figure 3.4 shows that the dynamic COLI is stationary. In other words, this index satisfies circularity or time reversal, an important property for the price index (see, for example, ILO et al. 2004). Among three time periods, $t_{0}, t_{1}$, and $t_{2}, \pi^{W}$ from $t_{0}$ to $t_{2}$ equals that from $t_{0}$ to $t_{1}$ times that from $t_{1}$ to $t_{2}$. Satisfaction of this property is obvious from equation (6), but not (8).

\subsection{Comparison with the Static COLI}

I compare the dynamic COLI with the static one. The static COLI is calculated using a standard model of perishable good and defined as a compensating variation to keep period utility, not welfare, constant. See the Appendix for details on the calculations. Table 1 shows that, when $p_{t}$ increases, the static COLI increases by 0.011. By the same size, it decreases when $p_{t}$ decreases. This magnitude is in the same order as that of the dynamic COLI based on definition (ii) (0.020). Further, both indexes exhibit no COLI change when $p_{t}$ is unchanged. Meanwhile, the comparison with the dynamic COLI based on definition (i) is not as obvious, because it has larger standard deviations and makes changes even when $p_{t}$ is unchanged.

Table 1: Comparisons of COLIs

\begin{tabular}{ccccc} 
& All & $\Delta \log \left(p_{t}\right)>0$ & $\Delta \log \left(p_{t}\right)=0$ & $\Delta \log \left(p_{t}\right)<0$ \\
\hline Dynamic COLI $\left(i_{t-1}\right)$ & $2.00 \mathrm{E}-06$ & -0.00137 & 0.00055 & -0.01797 \\
& $(0.0080)$ & $(0.0020)$ & $(0.0080)$ & $(0.0080)$ \\
Dynamic COLI $\left(i_{t-2}\right)$ & $-4.00 \mathrm{E}-06$ & 0.01960 & 0.00000 & -0.01973 \\
& $(0.0050)$ & $(0.0010)$ & $(0.0000)$ & $(0.0010)$ \\
Static COLI & 0.00000 & 0.01054 & 0.00000 & -0.01054 \\
& $(0.0020)$ & $(0.0000)$ & $(0.0000)$ & $(0.0000)$
\end{tabular}

Note: The figures represent the means of the changes in the COLIs generated by simulation. The figures in parentheses are standard deviations. COLI $\left(i_{t-1}\right)$ and COLI $\left(i_{t-2}\right)$ represents the dynamic COLI based on definition (i) and (ii), respectively. 

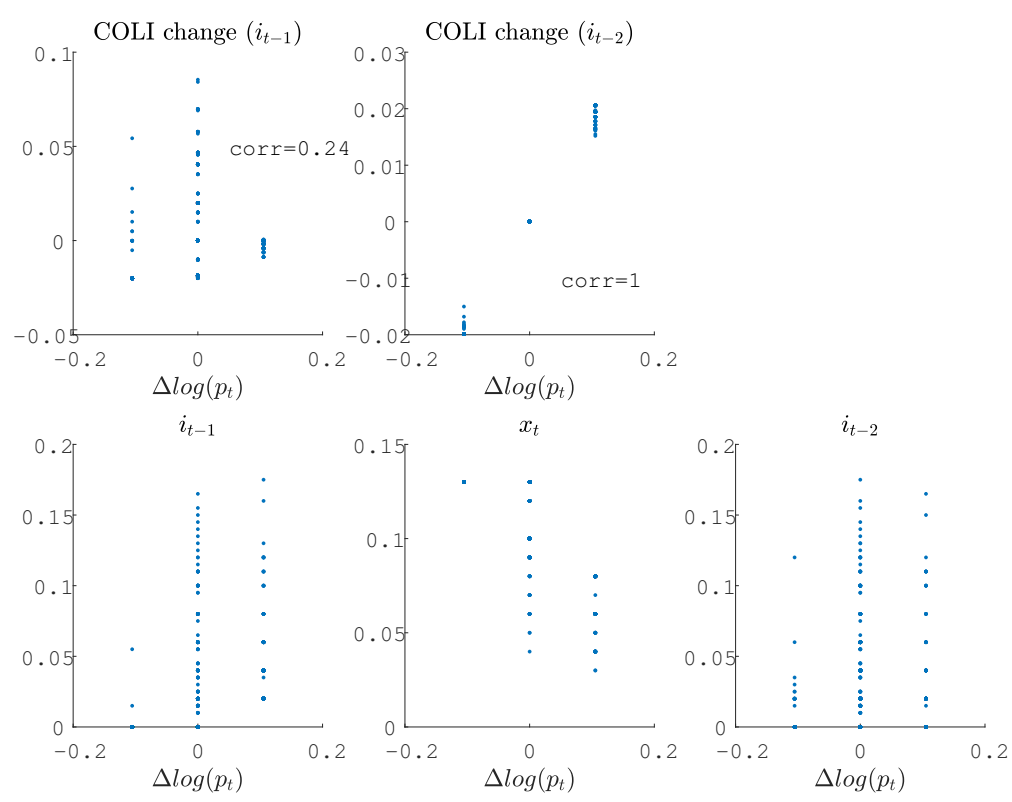

Figure 2: Change in COLI

Note: COLI $\left(i_{t-1}\right)$ and COLI $\left(i_{t-2}\right)$ represents the dynamic COLI based on definition (i) and (ii), respectively.

\subsection{Robustness}

I calculate how much monotonicity and time reversal change under different parameter values. Table 2 shows the simulation results. As for monotonicity, I report the correlation coefficient between the change in the COLI and the change in the price of storable goods. The correlation coefficient is always considerably lower than one, when the dynamic COLI is based on definition (i). Meanwhile, the correlation coefficient is one for both the dynamic COLI based on definition (ii) and the static COLI.

The time reversal test is satisfied for both the dynamic COLI based on definition (i) and the static COLI. However, again, the dynamic COLI based on definition (ii) does not satisfy this test. Furthermore, Table 2 shows that the size of the chain drift increases, when the effect of sales increases (i.e., $\bar{q}$ or $q$ increases or $p_{L} / p_{H}$ decreases). Specifically, when the size of sales discounts increases $\left(p_{L} / p_{H}\right.$ decreases from 0.9 to 0.8$)$, the size of deflation in the dynamic COLI based on definition (ii) increases from 3 percent in two decades to 42 percent. It is equivalent to 4 percent deflation annually, which is sizable.

\subsection{Dynamic COLI in a Regime Shift}

I examine how much the dynamic COLI changes when parameter values regarding sales change in a permanent and unanticipated manner. Before a regime change, I set $p_{L} / p_{H}=0.85$, 

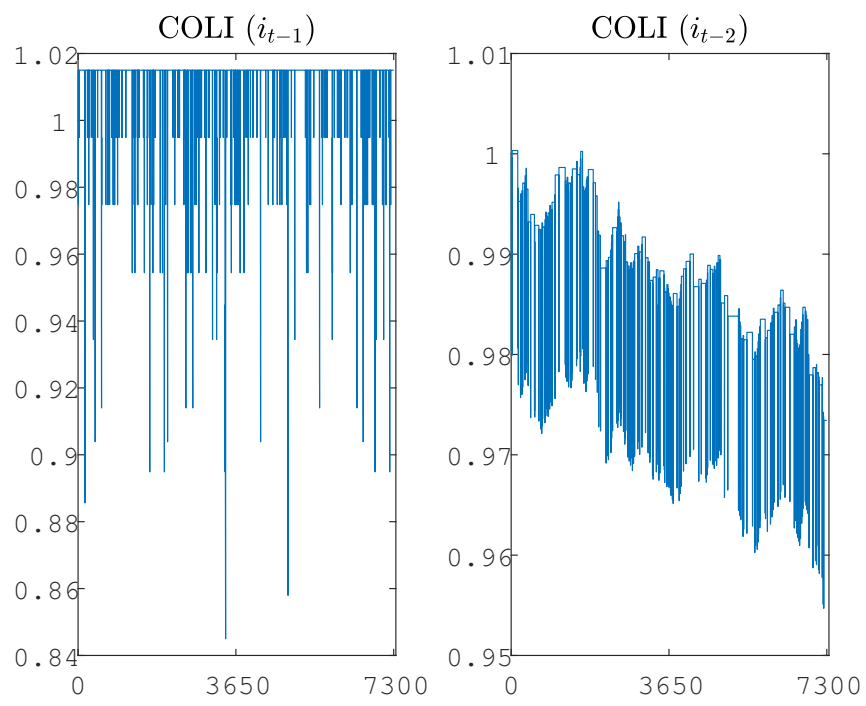

Figure 3: Movement of COLI

Note: COLI $\left(i_{t-1}\right)$ and COLI $\left(i_{t-2}\right)$ represents the dynamic COLI based on definition (i) and (ii), respectively.

$\bar{q}=0.017$, and $\underline{q}=0.600$. After the regime change, these values change to $p_{L} / p_{H}=0.90$, $\bar{q}=0.030$, and $q=0.500$, which are the same as in the above simulation. The values before the regime change corresponds to those in the 1990s according to Japanese retailers' scanner data, while those after the regime change correspond to the 2000s and 2010s. The frequency of sales has increased (higher $\bar{q}$ ) given $p_{t}=p_{H}$, but the duration of sales decreased (lower $\underline{q}$ ) and the size of sales discounts also decreased (higher $p_{L} / p_{H}$ ). The first result implies a price decrease, while the latter two imply a price increase.

I use definition (i) to compute the dynamic COLI. Definition (ii) cannot be applied here, because it suffers from the chain drift. I make a slight modification since the regime shift is permanent. The compensating variation $P_{t}^{W}$ is now assumed to be permanent:

$$
V\left(i_{t-1}, p_{t}, P_{t}^{W}\right)=V\left(i_{t-2}, p_{t-1}, 1\right)
$$

where $P_{t}^{W}$ enters into the second term on the right-hand side of the equation below:

$$
V\left(i_{t-1}, p_{t}, P_{t}^{W}\right) \equiv \max _{x_{t}, i_{t}}\left[u\left(i_{t-1}+x_{t}-i_{t}, P_{t}^{W}-p_{t} x_{t}\right)+\beta \mathbb{E}_{t} V\left(i_{t}, p_{t+1}, P_{t}^{W}\right)\right]
$$

In each regime, I generate the time series of prices for $T=365$, compute endogenous variables in each period, and compare the time series mean of $P_{t}^{W}$ between the two regimes. For comparison, I also consider the change in the static COLI. While the prices of storable goods take two 
Table 2: Robustness

(1) Monotonicity (correlation with price changes)

\begin{tabular}{cccc} 
& COLI $\left(i_{t-1}\right)$ & COLI $\left(i_{t-2}\right)$ & Static \\
\hline Benchmark & 0.24 & 1.00 & 1.00 \\
Higher $\bar{q}(0.05)$ & 0.10 & 1.00 & 1.00 \\
Higher $\underline{q}(0.75)$ & 0.36 & 1.00 & 1.00 \\
Lower $\bar{p}_{L}(0.8)$ & 0.20 & 0.99 & 1.00
\end{tabular}

(2) Time reversal (COLI changes from one in two decades)

\begin{tabular}{cccc} 
& COLI $\left(i_{t-1}\right)$ & COLI $\left(i_{t-2}\right)$ & Static \\
\hline Benchmark & 1.00 & 0.97 & 1.00 \\
Higher $\bar{q}(0.05)$ & 1.00 & 0.97 & 1.00 \\
Higher $\underline{q}(0.75)$ & 1.00 & 0.72 & 1.00 \\
Lower $\bar{p}_{L}(0.8)$ & 1.00 & 0.58 & 1.00
\end{tabular}

Note: Benchamrk specification is as follows: $\bar{q}=0.03, \underline{q}=0.50$, and $p_{L}=0.9$.

values, I compute the unconditional mean of period utility in each regime and its change after the regime changes.

Table 3 shows the results. At the regime change, the dynamic COLI increases by 0.0166 . To investigate the reason, I modify three parameter values one by one. When only $\bar{q}$ increases, the dynamic COLI decreases by 0.0043 . When only $\underline{q}$ decreases, the dynamic COLI increases by 0.0052 , which almost cancels out the previous decrease. Further, when $p_{L} / p_{H}$ increases, the dynamic COLI increases by 0.0166 . Overall, the dynamic COLI increases mainly due to the increase in $p_{L} / p_{H}$.

Meanwhile, the static COLI increases by 0.0001. The direction of the change is the same as that for the dynamic COLI, although the size of the change is smaller by the order of two. The decomposition of the change in the static COLI shows a similar pattern to that of the dynamic COLI. The increase in $\bar{q}$ decreases the static COLI, while the other two factors increase it.

Table 3: Changes in COLIs at Regime Change

\begin{tabular}{ccccc} 
& All & higher $\bar{q}$ & lower $\underline{q}$ & higher $p_{L} / p_{H}$ \\
\hline Dynamic COLI $\left(i_{t-1}\right)$ & 0.0166 & -0.0043 & 0.0052 & 0.0166 \\
Static COLI & 0.0001 & -0.0002 & 0.0001 & 0.0003
\end{tabular}

Note: Dynamic COLI $\left(i_{t-1}\right)$ is the dynamic COLI based on definition (i).

\section{Concluding Remarks}

No plausible dynamic COLI satisfies both monotonicity and the time reversal test. This poses a significant challenge to both economists and practitioners. Until we solve this problem, the use of the conventional static COLI may be better advised. 
Future research needs to search and propose desirable dynamic COLI that satisfies both monotonicity and the time reversal test. It also needs to be simple to understand and construct. For this, studies on consumer inventory are important from both empirical and theoretical perspectives.

\section{References}

[1] Boizot, Christine, Jean-Marc Robin and Michael Visser (2001) "The Demand for Food Products. An Analysis of Interpurchase Times and Purchased Quantities," Economic Journal, 111, 391-419.

[2] Erdem, Tulin, Susumu Imai, and Michael Keane (2003) "A Model of Consumer Brand and Quantity Choice Dynamics under Price Uncertainty," Quantitative Marketing and Economics, 1(1), 5-64.

[3] Feenstra, Robert, and Matthew Shapiro (2003) "High-Frequency Substitution and the Measurement of Price Indexes," in Scanner Data and Price Indexes, Robert Feenstra and Matthew Shapiro (eds.), University of Chicago and NBER.

[4] Gowrisankaran, Gautam, and Marc Rysman (2012) "Dynamics of Consumer Demand for New Durable Goods," Journal of Political Economy, 120, 1173-1219.

[5] Hendel, Igal, and Aviv Nevo (2006a) "Sales and Consumer Inventory." RAND Journal of Economics, 37, 543-561.

[6] Hendel, Igal, and Aviv Nevo (2006b) "Measuring the Implications of Sales and Consumer Inventory Behavior," Econometrica, 74, 1637-1673.

[7] ILO, IMF, OECD, Eurostat, United Nations, and World Bank (2004) "Consumer Price Index Manual: Theory and Practice," ILO Publications, Geneva.

[8] Ivancic, Lorraine, W. Erwin Diewert, and Kevin J. Fox (2011) "Scanner Data, Time Aggregation and the Construction of Price Indexes," Journal of Econometrics, 161, 2435.

[9] Könus A. A. (1924) "The Problem of the True Index of the Cost of Living," in Economic Bulletin of the Institute of Economic Conjuncture (in Russian), 9-10, 64-71; published in English (1939) in Econometrica, 7, 10-29. 
[10] Nakamura, Emi and Jon Steinsson (2010) "Monetary Non-Neutrality in a Multisector Menu Cost Model," Quarterly Journal of Economics, 125(3), 961-1013.

[11] Osborne, Matthew (2018) "Approximating the Cost-of-Living Index for a Storable Good," American Economic Journal: Microeconomics, 10(2), 286-314.

[12] Reis, Ricardo (2009) "A Dynamic Measure of Inflation," Working Paper.

[13] Sudo, Nao, Kozo Ueda, and Kota Watanabe (2014) "Micro Price Dynamics under Japan's Lost Decades," Asian Economic Policy Review, 9(1), 44-64.

[14] Sudo, Nao, Kozo Ueda, Kota Watanabe, and Tsutomu Watanabe (2018) "Working Less and Bargain Hunting More: Macroimplications of Sales during Japan's Lost Decades," Journal of Money, Credit and Banking, 50(2-3), 449-478.

[15] Wang, Emily Yucai (2013) "The Effect of More Accurate Cost-of-Living Indexes on Welfare Recipients," Working Paper.

\section{Appendix A. Model of Perishable Goods}

In a static model, there is no inventory, so $i_{t}=x_{t}$. In this study's specification, it follows $p_{t} x_{t}=\alpha$ and period utility and welfare equal

$$
u\left(p_{t}\right)=\alpha \log (\alpha)+(1-\alpha) \log (1-\alpha)-\alpha \log \left(p_{t}\right) .
$$

Compensating income, $P_{t}^{W}$, changes period utility by $\log \left(P_{t}^{W}\right)$; thus, the difference in $u\left(p_{L}\right)-$ $u\left(p_{H}\right)=\alpha \log \left(p_{H} / p_{L}\right)$ corresponds to $\log \left(P_{t}^{W}\right)$ when the price changes from $p_{H}$ to $p_{L}$, and vice versa.

We may consider a compensating income for welfare instead of period utility. Thus, we have

$$
\begin{gathered}
V\left(p_{t}\right)=\alpha \log (\alpha)+(1-\alpha) \log (1-\alpha)-\alpha \log \left(p_{t}\right)+\beta \mathbb{E}_{t} V\left(p_{t+1}\right), \\
V\left(p_{H}\right)=\alpha \log (\alpha)+(1-\alpha) \log (1-\alpha)-\alpha \log \left(p_{H}\right)+\beta\left\{(1-\bar{q}) V\left(p_{H}\right)+\bar{q} V\left(p_{L}\right)\right\} \\
V\left(p_{L}\right)=\alpha \log (\alpha)+(1-\alpha) \log (1-\alpha)-\alpha \log \left(p_{L}\right)+\beta\left\{(1-\underline{q}) V\left(p_{H}\right)+\underline{q} V\left(p_{L}\right)\right\},
\end{gathered}
$$


which leads to

$$
\left(\begin{array}{c}
V\left(p_{H}\right) \\
V\left(p_{L}\right)
\end{array}\right)=\left(\begin{array}{cc}
1-\beta(1-\bar{q}) & -\beta \bar{q} \\
-\beta(1-\underline{q}) & 1-\beta \underline{q}
\end{array}\right)^{-1}\left(\begin{array}{c}
\alpha \log (\alpha)+(1-\alpha) \log (1-\alpha)-\alpha \log \left(p_{H}\right) \\
\alpha \log (\alpha)+(1-\alpha) \log (1-\alpha)-\alpha \log \left(p_{L}\right)
\end{array}\right) .
$$

Compensating income, $P_{t}^{W}$, changes welfare by $\log \left(P_{t}^{W}\right)$; thus, the difference in $V\left(p_{L}\right)-V\left(p_{H}\right)$ corresponds to $\log \left(P_{t}^{W}\right)$ when the price changes from $p_{H}$ to $p_{L}$, and vice versa. 